\title{
Regularity Analysis for Patterned Texture Inspection
}

\author{
Henry Y. T. Ngan, Student Member, IEEE, and Grantham K. H. Pang, Senior Member, IEEE
}

\begin{abstract}
This paper considers regularity analysis for patterned texture material inspection. Patterned texture-like fabric is built on a repetitive unit of a pattern. Regularity is one of the most important features in many textures. In this paper, a new patterned texture inspection approach called the regular bands (RB) method is described. First, the properties of textures and the meaning of regularity measurements are presented. Next, traditional regularity analysis for patterned textures is introduced. Many traditional approaches such as co-occurrence matrices, autocorrelation, traditional image subtraction and hash function are based on the concept of periodicity. These approaches have been applied for image retrieval, image synthesis, and defect detection of patterned textures. In this paper, a new measure of periodicity for patterned textures is described. The Regular Bands method is based on the idea of periodicity. A detailed description of the RB method with definitions, procedures, and explanations is given. There is also a detailed evaluation using the Regular Bands of some patterned textures. Three kinds of patterned fabric samples are used in the evaluation and a high detection success rate is achieved. Finally, there is a discussion of the method and some conclusions.
\end{abstract}

Note to Practitioners-This paper is motivated by the study of a regularity feature for finding common properties in patterned textures. In general, regularity analysis of patterned textures involves two issues: the spatial relationship between intensity values and the repeat distance of a repetitive unit. These issues can also be defined as the periodicity of a patterned texture. However, the traditional periodicity is not effective for developing a patterned texture inspection algorithm. In this paper, a new measure for the regularity of patterned textures is designed for defect detection. It is based on the idea of applying the periodicity as a new principle for patterned texture inspection. A break in periodicity is considered to be a defect in patterned texture inspection. This concept has been applied to the development of a new method called the RB method. The regular band is defined by a moving average and standard deviation of the pixel intensity. It is specialized for defects which have differential intensity changes compared with the pattern on a repetitive unit of patterned texture. The RB method has been found very effective for defect detection of patterned fabric. In a comprehensive evaluation, the detection success rate of the $R B$ approach has reached $99.4 \%$ in a total of 166 defective and defect-free images taken from three patterned fabrics. In this paper, the techniques and detection results of the $R B$ method as well as comparisons with other methods are given. The computational time for processing an image of size $256 \times 256$ is only $140 \mathrm{~ms}$ using the $C$ programming language. This new approach for automated patterned texture inspection is believed to be useful for quality control. It will also make contributions not only to practitioners in the textile industry, but also in other industries like wallpaper and ceramics.

Manuscript received April 30, 2007; revised August 15, 2007. First published December 30, 2008; current version published December 30, 2008. This paper was recommended for publication by Associate Editor H. Qiao and Editor M. Wang upon evaluation of the reviewers' comments. This work was supported by Grants HKU 7169/03E and CRCG 10205131 from the University of Hong Kong.

The authors are with the Industrial Automation Research Laboratory, Department of Electrical and Electronic Engineering, University of Hong Kong, Pokfulam, Hong Kong (e-mail: ngan.henry@gmail.com; gpang@eee.hku.hk).

Digital Object Identifier 10.1109/TASE.2008.917140
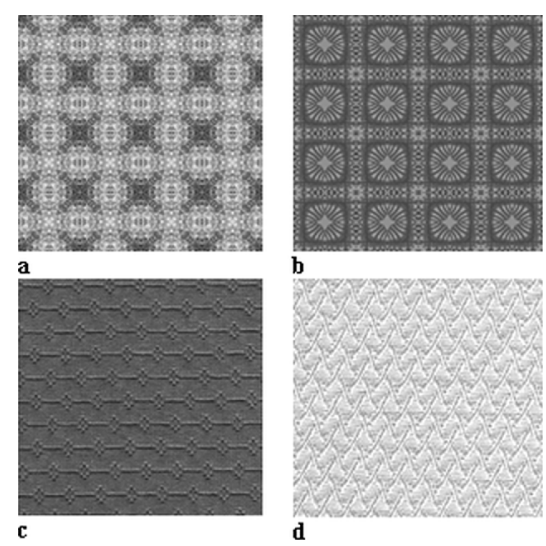

Fig. 1. (a), (b) Samples of simulated patterned textures. (c), (d) Samples of real patterned fabrics.

Index Terms-Fabric inspection, patterned texture analysis, periodicity, regular bands, regularity analysis.

\section{INTRODUCTION}

$\mathbf{T}$ EXTURE analysis has been studied by researchers for decades. In brief, textures can be broadly classified into patterned regular textures and irregular textures. This paper is focused on regularity analysis for patterned texture inspection. Textures can be interpreted in various ways in [1]-[3]. One viewpoint is to consider patterned textures to be constructed from a primitive (repetitive) pattern using a displacement rule. Then, texture analysis involves four main categories: texture segmentation, texture classification, texture synthesis and shape studies. Texture segmentation could be based on properties such as uniformity, density, coarseness, roughness, linearity, directionality, direction frequency, phase and regularity [4]. Among these properties, regularity analysis of patterned textures [5]-[10] has received growing attention. In general, regularity is an important feature because it is a highly invariant [5] but perceptually motivated feature. Hence, it is a good indicator of the randomness of a texture. A random texture such as a nature scene is considered to have minimal regularity. Otherwise, should there only be repetitive patterns in the texture (Fig. 1), it can be considered to be a patterned texture.

Regularity measurement usually involves the measurement of two features: the spatial relationship of intensity values and the repeat distance of repetitive units. For patterned texture analysis, previous regularity approaches like the methods of co-occurrence matrices [7], [8], autocorrelation [5], [6], directionality [5], [6], traditional image subtraction [11], [12] and the hash function [11], [13], [14], have been used in applications such as image retrieval, texture synthesis and defect detection. 

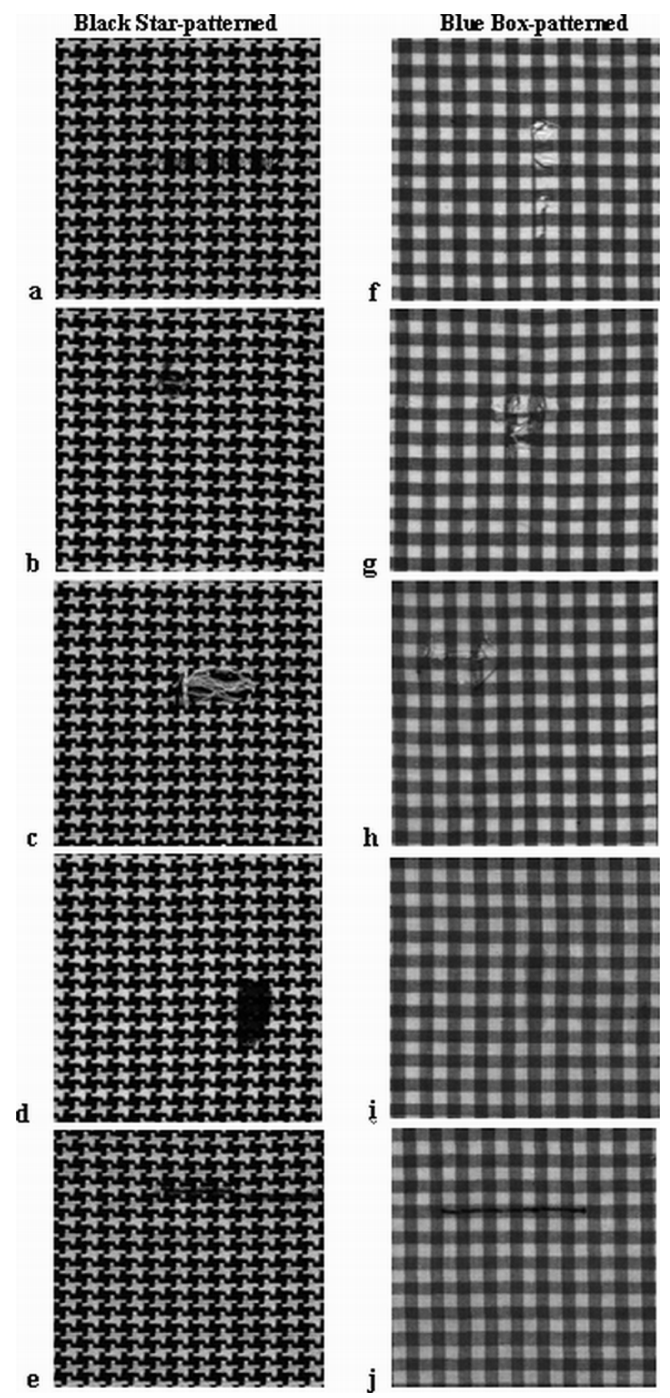

Fig. 2. Defective samples of (a), (f) broken end; (b), (g) hole; (c), (h) netting multiple; (d), (i) thick bar; and (e), (j) thin bar, in black star-patterned and blue box-patterned fabric.

Patterned texture defect detection is a key problem in quality control for many industrial fields like wallpaper scanning, ceramic flaw detection and fabric inspection. Among them, the textile industry is one of the biggest traditional industries that requires the development of an automated inspection system. Many techniques have been developed throughout the years using neural networks [15], [16], Fourier transform [17], [18], Gabor filters [19]-[21] and wavelet transform [22]-[24]. A good automated system means lower labor cost [25] and shorter production time [26]. However, most of the methods mentioned above are mainly designed for unpatterned fabric inspection. Patterned fabric inspection presents a difficult problem since the fabric has repetitive units on its design. For example, the defects may be relatively minor in comparison to those significant repetitive units, e.g., a broken yarn lying along the edge of a repetitive unit [Fig. 2(a)], or a think bar on several repetitive units [Fig. 2(i)].

There are several reasons that explain why researchers encounter difficulties in patterned fabric inspection. First, the texture complexity on the design of patterned fabric is much more sophisticated than for unpatterned fabric. This is because a patterned fabric image normally provides more underlying information on the spatial relationship among pixels than an unpatterned fabric image at the same level of resolution. Second, the categories of patterned texture are numerous. Two typical examples are shown in Fig. 1(c) and (d). Third, the similarity in shape between defects and background texture would be another obstacle for patterned fabric inspection. Fig. 2(a), (h), and (i), respectively, show defect samples of broken end of the star, netting multiple and thick bar of the box patterned fabrics, which are very similar to the repetitive units of the fabric.

In this paper, a star-patterned and box-patterned fabric database is used for defect detection. Every input patterned fabric sample is processed by histogram equalization in order to have a better contrast for defect detection. All images used in the evaluations are $256 \times 256$ pixels in grey level scale and the computation is carried out using MATLAB (version 7). In the first part of our evaluations, 106 images including 50 defect-free images and 56 defective images of two patterned fabrics are used. Fig. 2 shows five common types of defects (broken ends, holes, netting multiples, thick bars, thin bars) in fabric inspection.

The focus of this paper is on regularity approaches for patterned texture inspection. The main contributions of this paper are as follows.

1) A new approach based on the classical statistical method of moving average and standard deviation, which has been applied for patterned texture inspection.

2) Regular bands method is very effective for identifying defects with differential pixel intensity changes like thick bar and broken end.

3) Regular bands method is robust in design and needs only one parameter, the period length, for optimizing for the best result. Also, it can outline the defective region in the final images, which are clean and clear.

4) Defect detection result for three different fabric samples with 85 defect-free and 81 defective images is $99.4 \%$, which outperforms other previous methods.

The paper is organized as follows. First, a review of previous work is given in Section II. A new principle of periodicity for regularity analysis and the regular bands Method for patterned texture inspection are introduced in Section III. An evaluation using the regular bands method based on patterned fabrics is given in Section IV, including the detection results for box-patterned and star-patterned fabrics. Then, two recently developed methods, and the method of regular bands (RB) presented are compared for defect detection on a dot-patterned Jacquard fabric with 60 images. A comparison of the inspection results of these three methods are presented. More discussions are given in Section V. Finally, conclusions are delivered in Section VI.

\section{REVIEW OF PREVIOUS WORK}

\section{Definition of Regularity of Texture}

In previous research, the regularity of a patterned texture has two meanings: the spatial relationship between pixel intensities and the repeat distance of repetitive units. The spatial relationship between pixel intensities implies that one pixel in an image should have dependencies and steady changes with its 
surrounding neighborhoods on a patterned texture. The repeat distance of a repetitive unit is a measurement that can monitor whether the pattern distorts and overlaps within its placement rule for the construction of a whole image. This kind of regularity is related to the concept of periodicity.

\section{A. Previous Approaches for Patterned Texture Analysis}

1) Co-Occurrence Matrices: Co-occurrence matrices were designed by Haralick and Shapiro [27] to evaluate various properties of textures. They interpreted the spatial relationship between pixel intensities as fourteen different properties of co-occurrence matrices like entropy, energy, contrast, inertia, correlation, local homogeneity, dissimilarity and so on. The co-occurrence matrices approach has been applied to image retrieval [7] and defect detection [8] of patterned texture analysis since it is translational and rotational invariant [7]. However, high sensitivity to noise and heavy computational demand are two disadvantages of the co-occurrence matrices method. Furthermore, at least two pixel values in the designated spatial relationship are needed for calculation in every type of co-occurrence matrix.

2) Autocorrelation: Autocorrelation is a correlation between an image and its translated image. As a periodicity approach, it gives us information on the repeat distance of a repetitive unit in a patterned texture. Chetverikov et al. [5], [6] defined a contrast function between a polar representation of autocorrelation for an input image and the application of defect segmentation in unpatterned and patterned textures. Then, with the contrast function, a subcategory of periodicity, called directional regularity, was defined in [5] and [6]. This directional regularity was combined with two other regularity features, intensity regularity and position regularity. An outlier detection was then applied for defect detection. This regularity approach is very effective for linear and blob-like defects. Autocorrelation is an affine transformed invariant but it is also computationally expensive. This approach was tested in a few patterned texture examples of Brodatz and TILDA databases [6] but the results were not impressive.

3) Traditional Image Subtraction: The earliest approach for patterned fabric inspection was the traditional image subtraction (TIS) method. Chin and Harlow [25] tried to use an exclusive-OR (XOR) operation as the image subtraction method for the traditional defect inspection of printed circuit boards (PCBs). This involves the subtraction of an entire image of the test circuit board from a perfect master image of the same type of circuit board. Obviously, the TIS method was using the information of repeat distance of a repetitive unit as well as the periodicity in patterned textures. Later, Tao et al. [28] and Sandy et al. [12] suggested using the same technique as [25] on lace, which is one kind of patterned fabric with fine and complex thread patterns. The subtractions on lace are based on a prototype version and a test version of the same pattern. The procedures of the TIS method are as follows: partitioning the input test image, converting the grey level image to a binary image, XOR operation between the reference image (also called the golden image) and the test image, and smooth filtering. The TIS method is sensitive to noise because an input image is easily distorted and it is hard to do a point-to-point comparison. Dot-patterned Jacquard fabric inspection using the TIS method was also found to be unsuccessful in [11].
4) Hash Function: Another method which can be applied to patterned texture inspection is based on the hash function. The hash function originated from cryptographic studies for data encryption and decryption, and it is sensitive to any small changes during data transmission. Baykal et al. [13], [14] transferred this property and interpreted it for defect detection of patterned textures. There are four basic families of hash functions [13]: checksum, plain, XOR, and multiplication. The choice of hash function type depends on the complexity of the texture. There exist offset effects for calculating the hash functions among regular (defect-free) patterns. It uses the spatial relationship between two neighboring pixels. For more complex patterned textures, high order hash functions such as XOR and multiplication are chosen. On the other hand, the checksum hash function is just defined as the sum of all pixel values on each horizontal line, but it does not give much information on small defects of large complex patterned fabrics. The advantage of this method is that it is time-saving due to its one-dimensional approach. The disadvantage is its sensitivity to noise and its inability to outline the shape of the defect after detection. A preliminary study to apply the hash function method on dot-patterned Jacquard fabric was conducted in [11] but the results were poor.

5) Near-Infrared (NIR) Imaging Method: The NIR method [29] is a hardware approach to utilize near-infrared (NIR) illumination instead of the traditional visible (VIS) light source for patterned fabric defect detection. The defects are usually undistinguishable in the usual VIS image. Millan and Escofet [29] defines the basic patterned fabric structure as low energy structure signal where squares, circles and design pattern as high energy signal. The high energy signal usually constitutes noises which lead to the failure of detection. However, the NIR illumination transforms the input image into a new NIR image that severely reduce the high energy signal. Then, by thresholding, the defects like holes and broken ends can be detected.

\section{B. Brief Review of Previous Approaches to Periodicity With a New Principle}

Three new methods for patterned fabric inspection, the wavelet preprocessed golden image subtraction (WGIS) method [30] the method of direct thresholding (DT) for detailed subimages [11], and the Bollinger bands (BB) method [31] have recently been developed. These three methods are based on the new principle of measuring the periodicity of the input images. The key concepts are introduced as follows.

1) Golden Image Subtraction Method: The basic golden image subtraction (GIS) method [30] is designed for detecting defects of patterned fabrics and the WGIS is an improved version of it. The GIS method is different from that of Chin and Harlow [25] since a golden image can contain several repetitive units taken from a defect-free image. In addition, the golden image used in GIS, performing like a convolution filter, slides on the test image, and it is not a static comparison between the golden template and the test image. The GIS method can highlight the defective region after thresholding on the image of energies of GIS [Fig. 3(e) and (g)] for every input image. The GIS method is shift-invariant and the main formula is shown below. 


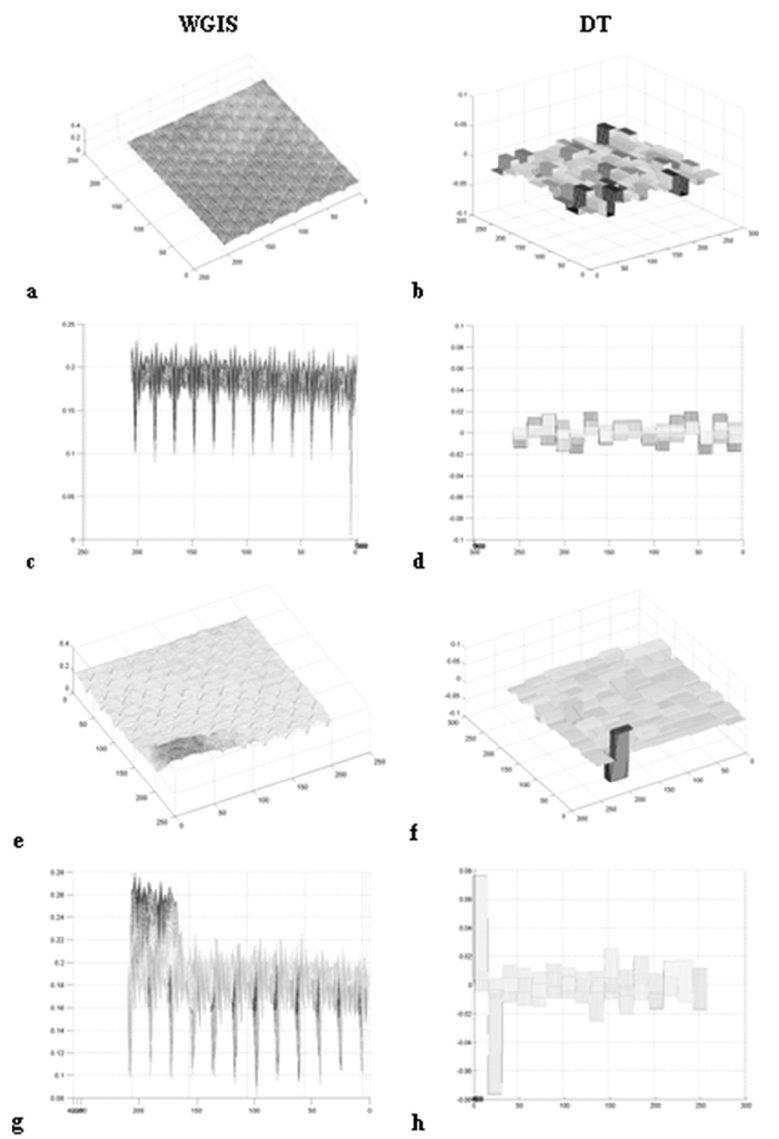

Fig. 3. (a), (b) Perspective plots; (c), (d) radial cross sections of the energies of GIS and fourth level vertical subimage of Haar wavelet decomposition on a defect-free image. (e), (f) Perspective plots, (g), (h) radial cross sections of the energies of GIS and the fourth level vertical subimage of Haar wavelet decomposition on a defective image, thin bar in Fig. 12(f).

Let a golden image be $G=\left(g_{i j}\right)$ with a size of $a \times b$ pixels and a subtracted image $H=\left(h_{i j}\right)$ with a size of $a \times b$ pixels. The size of every input image is $p \times q$. Then, the energy of GIS is defined as

$$
R=\left(r_{x y}\right)
$$

where $r_{x y}=(1 /(a \cdot b)) \sum_{i=1}^{a} \sum_{j=1}^{b}\left|g_{i j}-h_{i j}\right|, x=$ $1, \ldots, p-a+1$ and $y=1, \ldots, q-b+1(0<b \leq q$, $0<a \leq p)$.

2) Direct Thresholding Method: In the method of direct thresholding for detailed subimages [11], the multiresolution theory of wavelets is applied. Every input image $F(x, y)$ at level $m$ size of $2^{-m} \times 2^{-m}$ would undergo the Haar wavelet transform for extracting detailed subimages at the fourth level. Suppose $\Phi$ to be a scaling function and $\Psi$ to be the corresponding wavelet function for a two-dimensional view. Let $\psi$ and $\phi$, respectively, be the one-dimensional wavelet and scaling functions. Also, let the $L$ and $H$ subscripts, respectively, denote the low-pass and high-pass functions.

The key formulas are shown

$$
\begin{aligned}
& F_{L L}(x, y)=\phi_{x} \phi_{y}(F(x, y)) \\
& F_{L H}(x, y)=\phi_{x} \psi_{y}(F(x, y)) \\
& F_{H L}(x, y)=\psi_{x} \phi_{y}(F(x, y)) \\
& F_{H H}(x, y)=\psi_{x} \psi_{y}(F(x, y)) .
\end{aligned}
$$

By the equations above, an input image $F(x, y)$ is decomposed into four subimages where $F_{L L}(x, y), F_{L H}(x, y), F_{H L}(x, y)$, and $F_{H H}(x, y)$ denote the coarse approximation, horizontal detailed, vertical detailed and diagonal detailed subimages of the image for a one level decomposition at a resolution $2^{-(m+1)} \times$ $2^{-(m+1)}$

In the direct thresholding method, the Haar wavelet coefficients are employed and the length of the decomposition filter is two. It is sufficient to obtain good detailed subimages to enhance the defective regions in a defective image [Fig. 3(f) and (h)]. Defects usually appear as high frequency elements in the defective image. A wavelet transform of the DT method is used to enhance the defective information in the horizontal and vertical directions.

3) Bollinger Bands (BB) Method: The Bollinger bands, constitutes of three bands, are originally applied on the stock market as an one-dimensional indicator for oversold and overbought trends. Bollinger bands are calculated based on two statistical tools, moving average and standard deviation. Ngan and Pang [31] extended BB from a one-dimensional approach into a twodimensional approach for patterned Jacquard fabric defect detection. The formulas of the BB method are as follows.

For a particular row in an image $X=\left(x_{i j}\right)$ of size $p \times q$

Middle band : $M_{r_{n}}=\frac{\left(\sum_{j=r_{1}}^{r_{n}} x_{i j}\right)}{n}$

$$
\text { Upper band }: B_{r_{n}}^{U}=M_{r_{n}}+\left\{d \cdot \sqrt{\frac{\left\{\sum_{j=r_{1}}^{r_{n}}\left(x_{i j}-M_{r_{n}}\right)^{2}\right\}}{n}}\right\}
$$

$$
\text { and Lower band: } B_{r_{n}}^{L}=M_{r_{n}}-\left\{d \cdot \sqrt{\frac{\left\{\sum_{j=r_{1}}^{r_{n}}\left(x_{i j}-M_{r_{n}}\right)^{2}\right\}}{n}}\right\}
$$

where $U$ indicating the Upper band and $L$ indicating the Lower band. $d$ denotes the number of standard deviation and $n$ denotes the row dimension of the repetitive unit. $x_{i j}$ is the pixel value at row $i$, column $j$ of the image $X$, the summation is from $r_{1}^{\text {st }}$ pixel to $r_{n}^{\text {th }}$ pixel with $1 \leq r_{1} \leq r_{n} \leq q, r_{n}=r_{1}+n, r_{1} \in[1, q-n]$, $r_{n} \in[1+n, q]$ and $B_{r_{n}}^{U}, B_{r_{n}}^{L}, M_{r_{n}} \in R$.

The upper and lower Bollinger bands can accentuate the defective information after calculation and attenuate the background information of patterned texture. The BB method requires tuning of two parameters, $n$ and $d$, in order to achieve for the best detection result.

\section{NeW MEASUREMENT OF REgUlarity FOR PATTERNED TEXTURES}

\section{A. New Principle of Measurement for Regularity}

A repetitive unit is the fundamental component of a patterned texture, which can be broadly viewed as either one-dimensional 

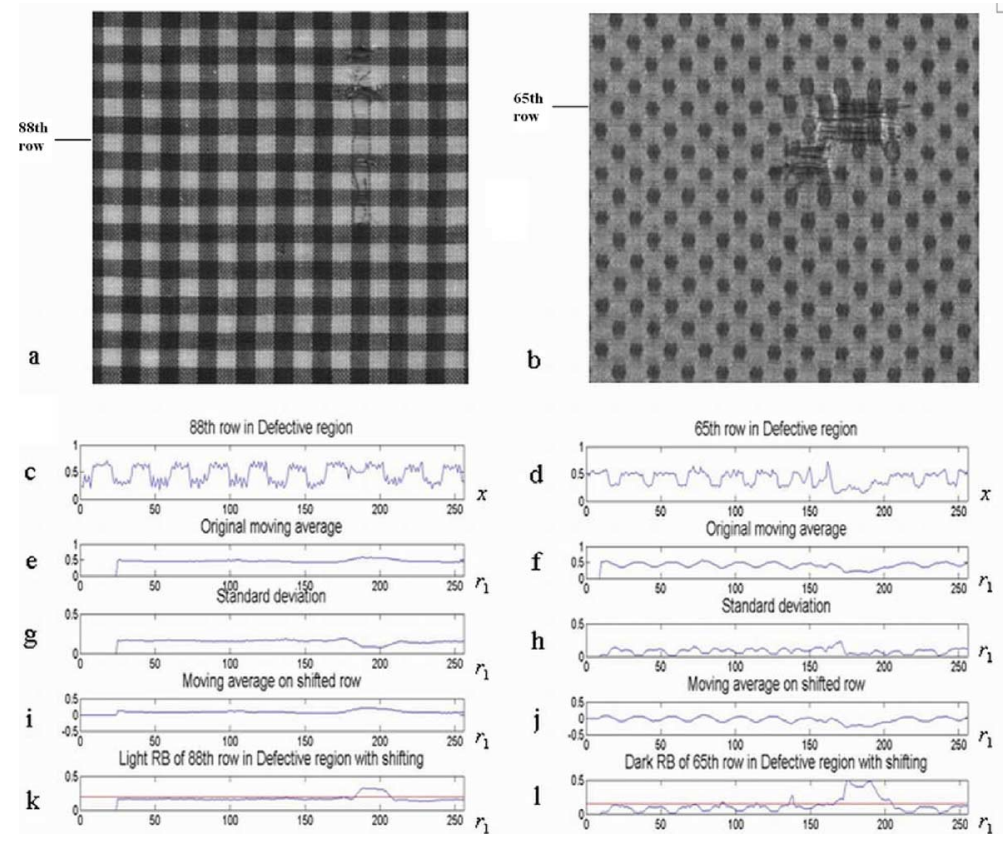

Fig. 4. (a) Defective box-patterned fabric sample (c) the plots of the 88th row, (e) the moving average of the 88th row, (g) the standard deviation of the 88th row, (i) the moving average of the shifted 88th row, and (k) LRB on the 88th row. (b) Defective circle-patterned fabric sample, (d) the plots of the 65th row, (f) the moving average of the 65th row, (h) the standard deviation of the 65th row, (j) the moving average of the shifted 65th row, and (l) DRB on the 65th row, respectively $r_{1} \in[1, q-n]$. (Lines are marking the threshold value of the upper bound of the range of one standard deviation WITH shifting in preprocessing step.)

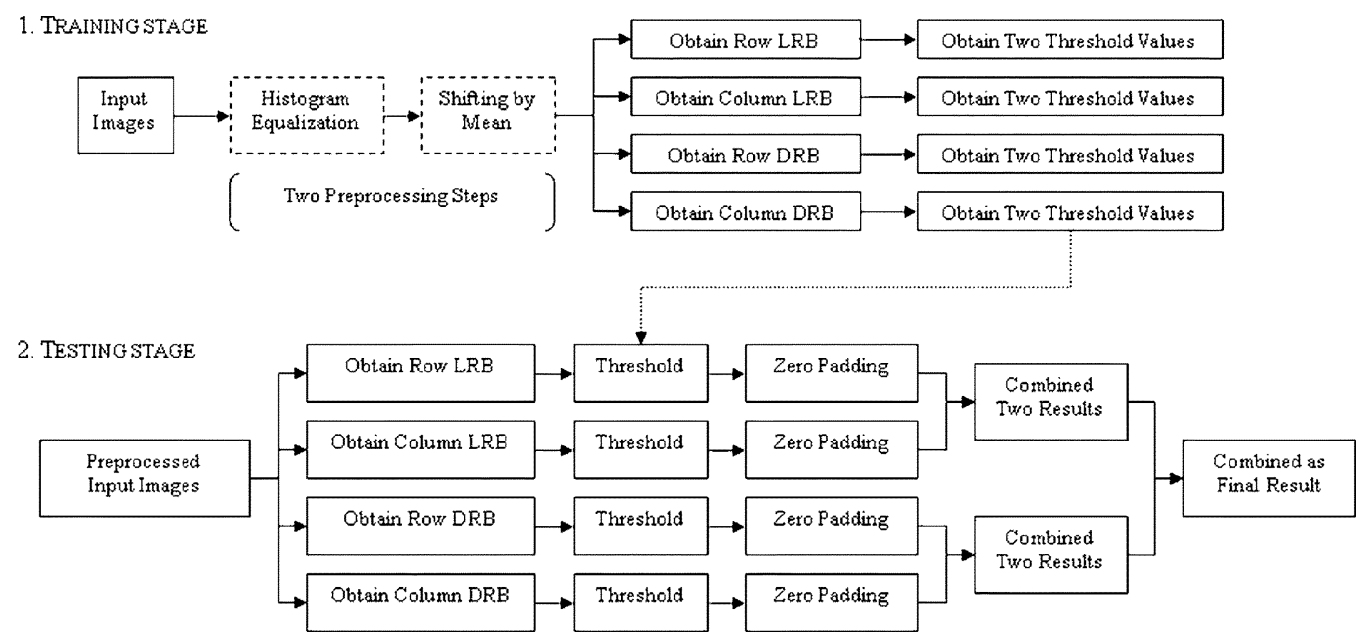

Fig. 5. Stages of regular bands method.

(e.g., periodic signal) or two-dimensional (e.g., patterned image). For example, in an one-dimensional view, applying an appropriate transformation to the normal parts (defect-free) of a periodic signal can result in its explicit expression as an intuitive periodicity, which is defined as a new kind of regularity of the patterned texture. This transformation is constructed by using the structural characteristics of the repetitive unit of any patterned texture. In other words, the structural characteristic is obtained by using the repetitive unit as a convolution filter sliding on the test signal. The numerical values of an abnormal part (defective region) would exceed the normal range of the signal. Therefore, by designing a suitable transformation, any numerical values of the abnormal part is significant enough to be segmented out using thresholding and the shape of any defective region can be outlined.

\section{B. Regular Bands (RB) Method}

The RB consists of two subbands, the light regular band (LRB) and the dark regular band (DRB). The method of RB mainly consists of two stages: a training stage and a testing stage. The definitions, descriptions and physical meanings of LRB and DRB are delivered as follows.

1) Preprocessing Step for Every Input Image: First, a collection of $f$ defect-free images of size $p \times q$ is obtained, $Y_{k}=\left(y_{i j}^{k}\right)$ where $k=1, \ldots, f, i=1, \ldots, p$ and $j=1, \ldots, q$. Second, a mean value, $y_{k}=\operatorname{mean}\left(y_{i j}^{k}\right)$, of every defect-free sample is calculated, and a mean of all $y_{k}$. $\left\{\right.$ i.e., $\left.\bar{y}=\operatorname{mean}\left(y_{k}\right)\right\}$ is obtained. Lastly, let $\bar{X}_{b}=\left(\bar{x}_{i j}^{b}\right)$ be every input image of an image database with $h$ images, where $b=1, \ldots, h, i=1, \ldots, p$ and $j=1, \ldots, q$. A down-shifting of all pixel values by $\bar{y}$ is made 
Defect-free

Row LRB of Defect-free 1

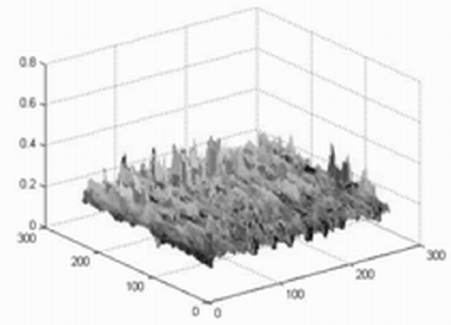

Row LRB of Defect-free 1
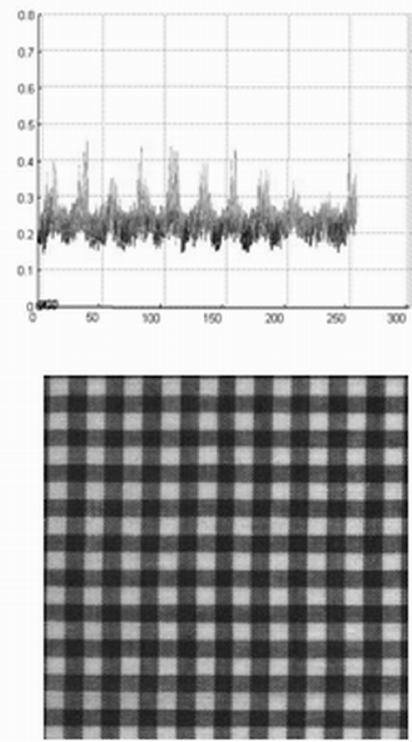

Defective

Row LRB of Broken End 1

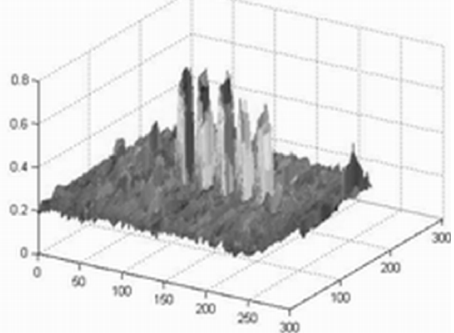

b

Row LRB of Broken End 1

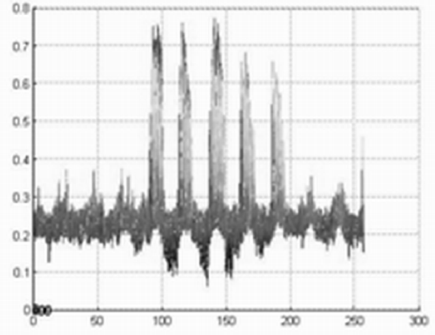

d

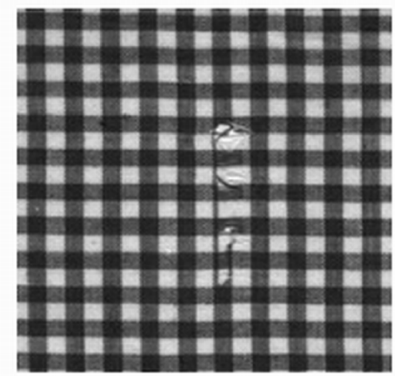

Fig. 6. (a), (b) Perspective plots and (c), (d) radial cross sections after calculating LRB on rows for (e) a defect-free sample, (f) a defective broken end sample on box-patterned fabric, respectively.
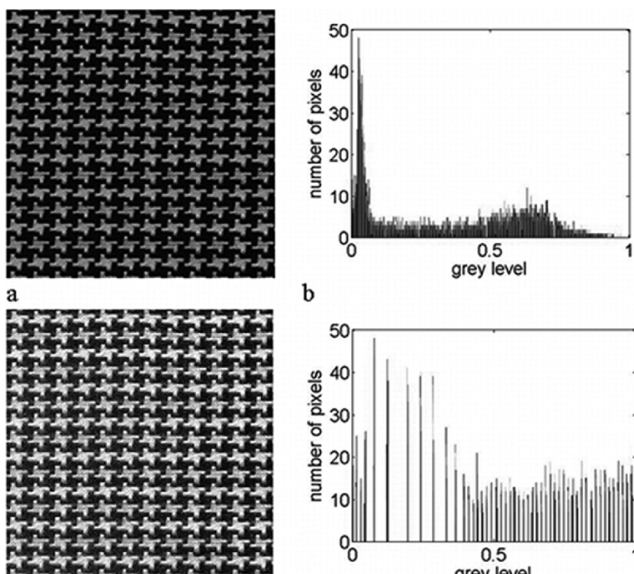

$\mathrm{c}$

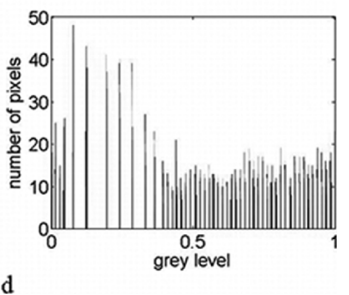

Fig. 7. (a) Defect-free sample of star-patterned fabric without histogram equalization, (b) the histogram of (a), (c) defect-free sample of Star-patterned fabric with histogram equalization, (d) the histogram of (c).

to obtain a renewed database $\left\{\right.$ i.e., $\left.X_{b}=\left(\bar{x}_{i j}^{b}-\bar{y}\right)=\left(x_{i j}^{b}\right)\right\}$, where $x_{i j}^{b} \in[0-\bar{y}, 1-\bar{y}]$ and $\bar{x}_{i j}^{b} \in[0,1]$.
Definition of Regular Bands: For a particular row in a preprocessed image from the renewed database, $X=\left(x_{i j}\right)$, of size $p \times q$.

The light regular band (LRB) is defined as

$$
L_{r_{n}}=\left|u_{r_{n}}-\sigma_{r_{n}}\right|+u_{r_{n}} .
$$

The dark regular band (DRB) is defined as

$$
D_{r_{n}}=\left|u_{r_{n}}+\sigma_{r_{n}}\right|-u_{r_{n}}
$$

where the moving average $u_{r_{n}}$ is defined as

$$
u_{r_{n}}=\frac{\left(\sum_{j=r_{1}}^{r_{n}} x_{i j}\right)}{n}
$$

where $n$ is an integer value denoting the row dimension of the repetitive unit and $x_{i j}$ is the pixel value at row $i$, column $j$ of the image $X$, the summation is from $r_{1}^{\text {st }}$ pixel to $r_{n}^{\text {th }}$ pixel with $1 \leq r_{1} \leq r_{n} \leq q, r_{n}=r_{1}+n, r_{1} \in[1, q-n], r_{n} \in[1+n, q]$, and $L_{r_{n}}, D_{r_{n}}, u_{r_{n}} \in R$. 

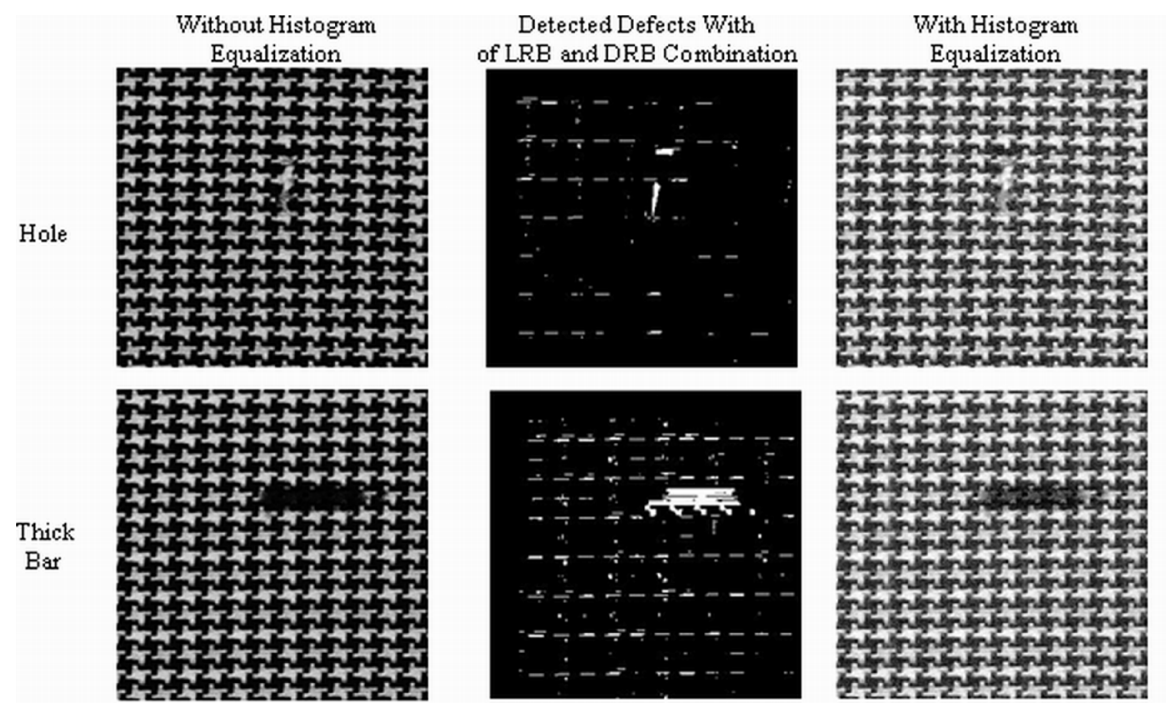

Detected Defects With of LRB and DRB Combination
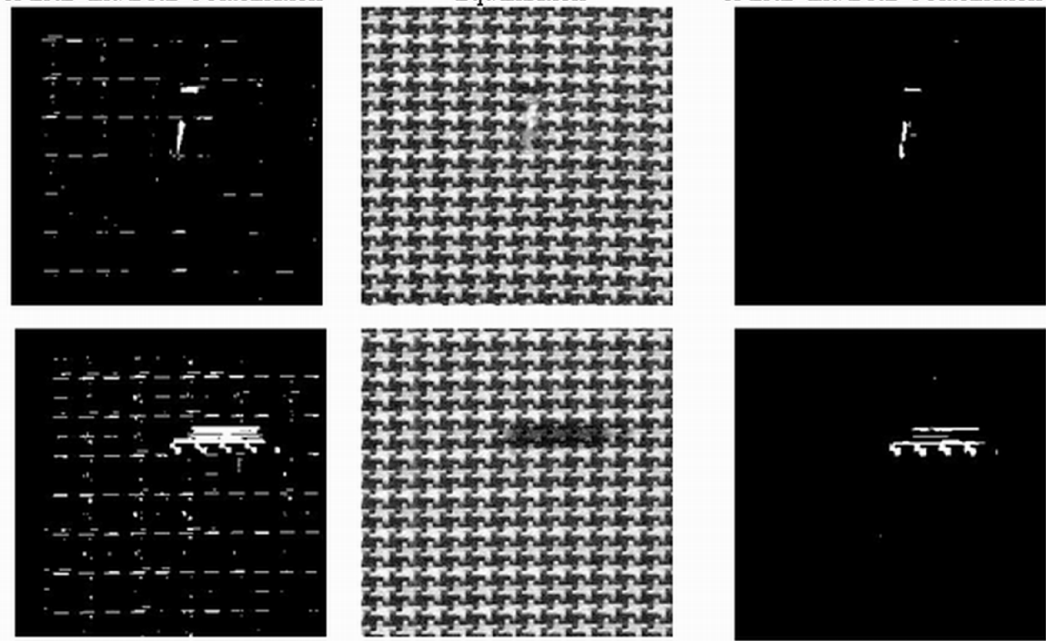

Fig. 8. Detection results of two defective samples, hole and thick bar, with and without preprocessed histogram equalization.
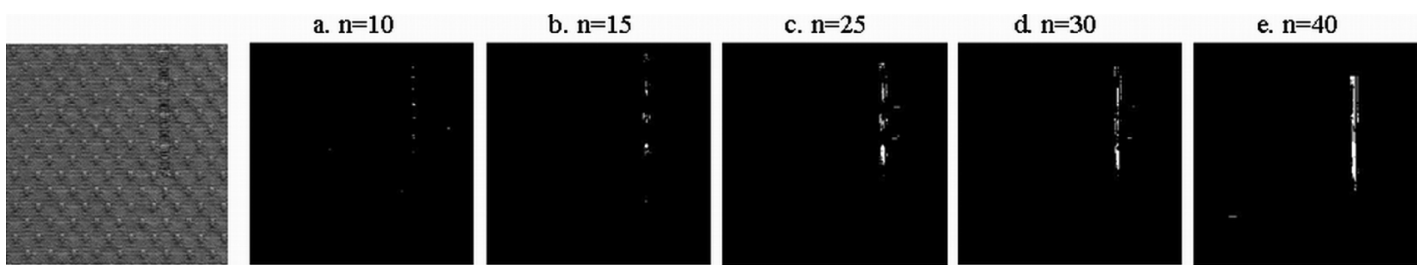

Fig. 9. Broken-end sample of dot-patterned fabric and the detection results of different numbers of period, (a) $n=10$, (b) $n=15$, (c) $n=25$, (d) $n=30$, and (e) $n=40$.

The standard deviation is defined as

$$
\sigma_{r_{n}}=\sqrt{\frac{\left[\sum_{j=r_{1}}^{r_{n}}\left(x_{i j}-u_{r_{n}}\right)^{2}\right]}{n} .}
$$

The nominal value for $n$ is twenty-five, which is the row dimension of a repetitive unit of black star-patterned and blue box-patterned fabrics under evaluation in this paper. The value of $n$ is affected by the resolution of the input image.

2) Meanings of RB: Regular Bands are used to detect defects using a regularity approach. The key idea of the regularity approach is to study or represent signal generation for each vertical and horizontal line of the defect-free region. Any defect in a defective region would correspond to an irregularity in the signal. In this method, the moving average and standard deviation are a pair of tools to achieve the desired performance.

Standard deviation is a very good measure for indicating any irregularity of a signal. The maximum and minimum values of the standard deviation of a signal form a range which is a good tolerance for normal variations of the regular signal. A defective part is believed to exceed the range of the regular signal, which is set as one standard deviation.

In fabric inspection, the boundaries between defective and defect-free regions are normally considered high frequency irregular changes, especially for defects such as broken end, hole, and thick bar. Broken end [Fig. 2(f)] and thick bar [Fig. 2(d)] are typical types of defects that are lighter and darker in color, respectively, compared with the background patterns that also provide useful defective information for defect detection.

Need of Down-Shifting of Image: Before introducing the regular bands, there is a need to explain the mathematical formulas embedded in them. In the regular bands method, a preprocessing step of downshifting the pixel intensities of each input image is taken. The aim of this shifting pixel intensity is to make a sequence of pixel intensities $X_{k}$, where $k=1, \ldots, f$, which range from negative to positive on the real plane, in order to fulfill the mathematical design of regular bands. The original pixel intensities are in the range from 0 to 1 . The LRB and DRB are motivated by this treatment and discussed as below.

Motivation of $L R B$ and DRB: The following describes the reasoning behind the use of the light regular band and dark regular band. Fig. 4 depicts how the LRB and DRB tackle the problem using classical statistical tools.

To Deal With Light Defects: Light Regular Band: For example, suppose that the goal is to detect the defect on the 88th row [Fig. 4(c)] somewhere between the 170th and 200th pixels. The original moving average $\left(u_{r_{n}}\right)$ is shown in Fig. 4(e) and the standard deviation $\left(\sigma_{r_{n}}\right)$ is shown in Fig. $4(\mathrm{~g})$. Notice that at the defective region, the values of $\sigma_{r_{n}}$ do not exceed the normal range of one standard deviation (threshold), and hence fail to indicate the light defect. Denoting the moving average of the shifted 88th row in Fig. 4(i), $u_{r_{n}}$ should be around zero at the nondefective region. However, at the defective region $u_{r_{n}}$ is 


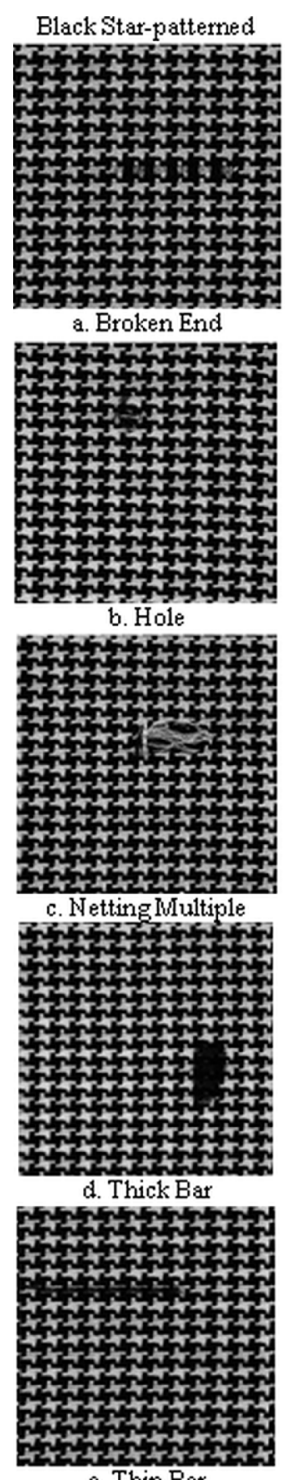

e. Thin Bar
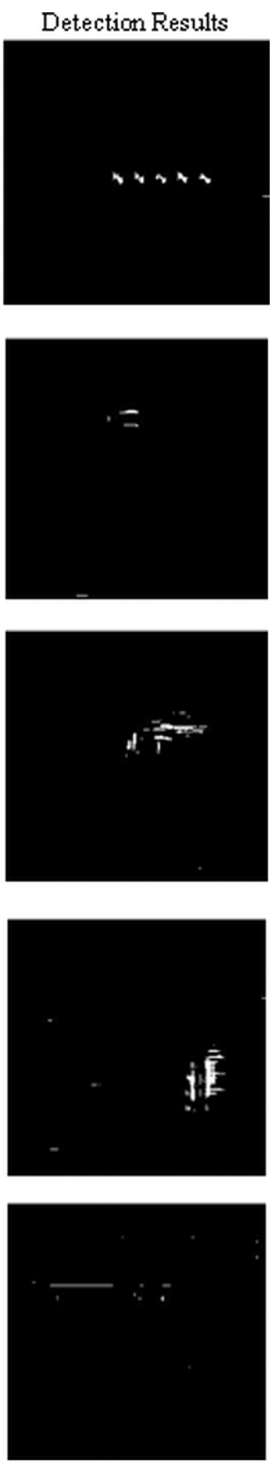

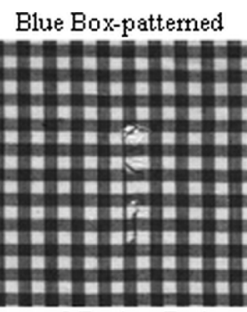

f. Broken End

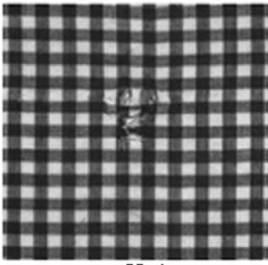

g. Hole

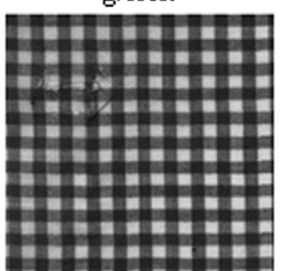

h. Netting Multiple

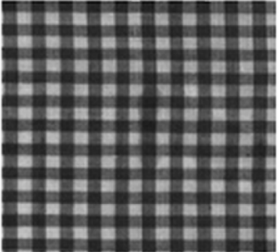

i. Thick Bar

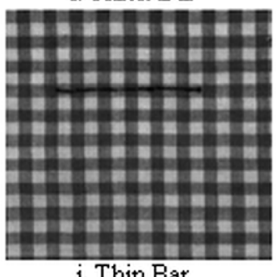

j. Thin Bar
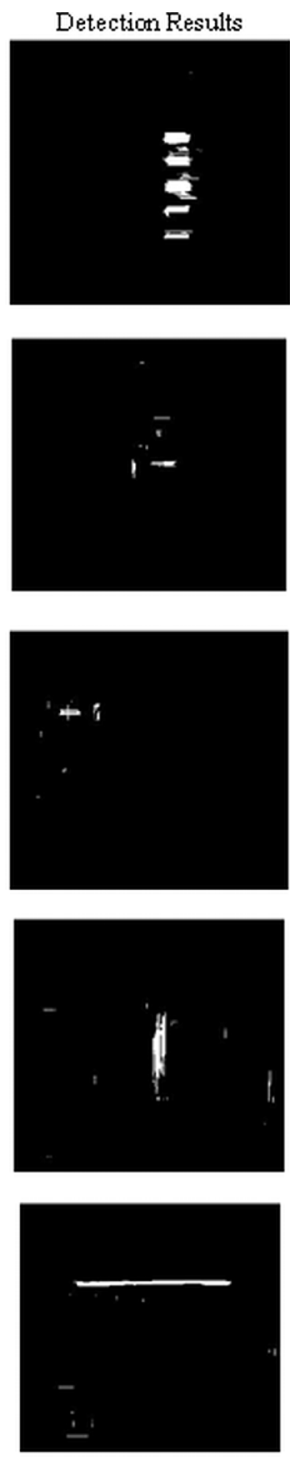

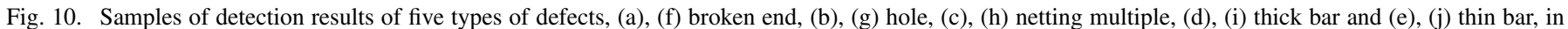
star-patterned fabric and box-patterned fabric, respectively.

higher than zero and $\sigma_{r_{n}}$ is lower than normal. Therefore, a measure $(M)$ for the severity of the defect should be $\left|u_{r_{n}}-\sigma_{r_{n}}\right|+K$ where $K$ is a constant to be determined. That is

$$
M=\left|u_{r_{n}}-\sigma_{r_{n}}\right|+K .
$$

At the nondefective region, we want $M=\sigma_{r_{n}}$. With $u_{r_{n}}$ around zero and $\sigma_{r_{n}}$ nonzero, we should choose $K$ equal to $u_{r_{n}}$, so that the measure $M$ is equal to $\sigma_{r_{n}}$.

At the defect region, $u_{r_{n}}>0$ and $\sigma_{r_{n}}$ is small

$$
M=\left|u_{r_{n}}-\sigma_{r_{n}}\right|+u_{r_{n}} .
$$

If $u_{r_{n}} \geq \sigma_{r_{n}}, M=2 u_{r_{n}}-\sigma_{r_{n}}$, and this value should exceed a normal value of the standard deviation, thus indicating the defect [Fig. 4(k)]. If $u_{r_{n}}<\sigma_{r_{n}}, M=\sigma_{r_{n}}$, and this measure would use one standard deviation as the measure for defects.

To Deal With Dark Defects: Dark Regular Band: Similarly, we wish to detect the defect in Fig. 4(b), which is on the 65th row between the 130 th and 225 th pixels. Fig. 4(f) presents the original moving average $\left(u_{r_{n}}\right)$ and Fig. 4(h) displays the standard deviation $\left(\sigma_{r_{n}}\right)$. Upon closer inspection, the values of $\sigma_{r_{n}}$ at the defective region do not exceed the normal range of standard deviation. Therefore, the dark defect there is not detected. In Fig. 4(j), $u_{r_{n}}$ should be around zero at the nondefective region. However, $u_{r_{n}}$ is less than zero and $\sigma_{r_{n}}$ is smaller than normal in the defective region. For a severity of the defect, a measure $(M)$ should be $\left|u_{r_{n}}+\sigma_{r_{n}}\right|-K$ where $K$ is a constant to be determined. That is, $M=\left|u_{r_{n}}+\sigma_{r_{n}}\right|-K$.

At the nondefective region, we want $M=\sigma_{r_{n}}$, similar to the design of the LRB. We should choose $K$ to be equal to $u_{r_{n}}$ under the condition that $u_{r_{n}}$ is sufficiently close to zero and $\sigma_{r_{n}}$ is nonzero. Hence, the value of $M$ is equal to $\sigma_{r_{n}}$.

At the defective region, $u_{r_{n}}<0$ and $\sigma_{r_{n}}$ is small

$$
M=\left|u_{r_{n}}+\sigma_{r_{n}}\right|-u_{r_{n}} .
$$

As a result, if $u_{r_{n}} \geq \sigma_{r_{n}}$ then $M=2 u_{r_{n}}-\sigma_{r_{n}}$. To identify the defect, this value should exceed a normal value of the stan- 
Final Results of Methods for Defect Detection on Dot-patterned Jacquard Fabric on Different Defective Images

(a) Broken End, (b) Holes, (c) Knots, (d) Netting Multiple, (e) Thick Bar, (f) Thin Bar

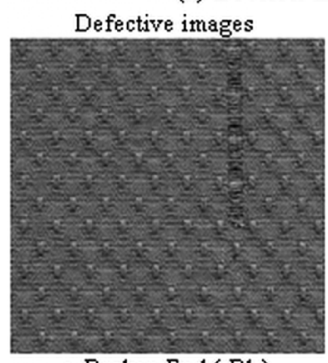

a. Broken End ( B1 )

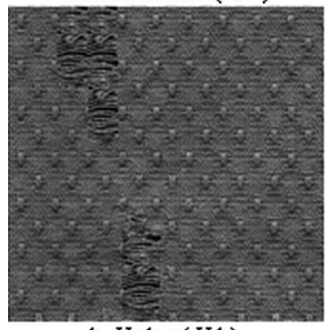

b. Holes ( $\mathrm{H} 1$ )

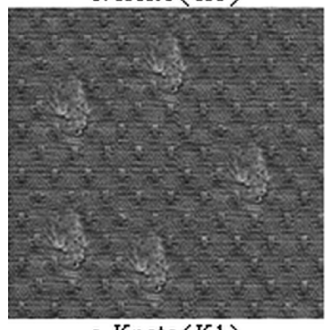

c. Knots (K1)

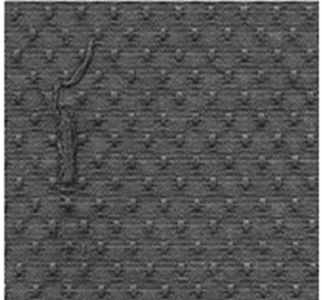

d. Netting Multiple (N1)

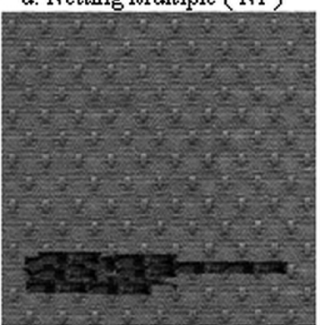

e. Thick Bar (Tk1)

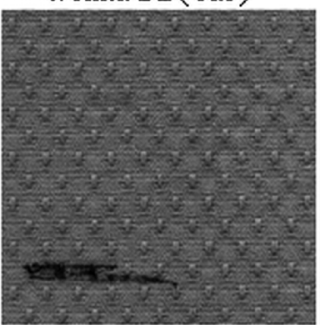

f. Thin Bar ( Tn1 )
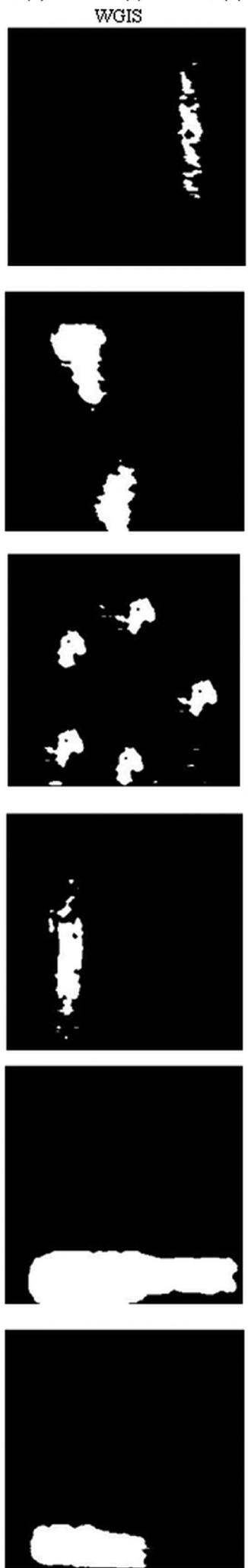

DT
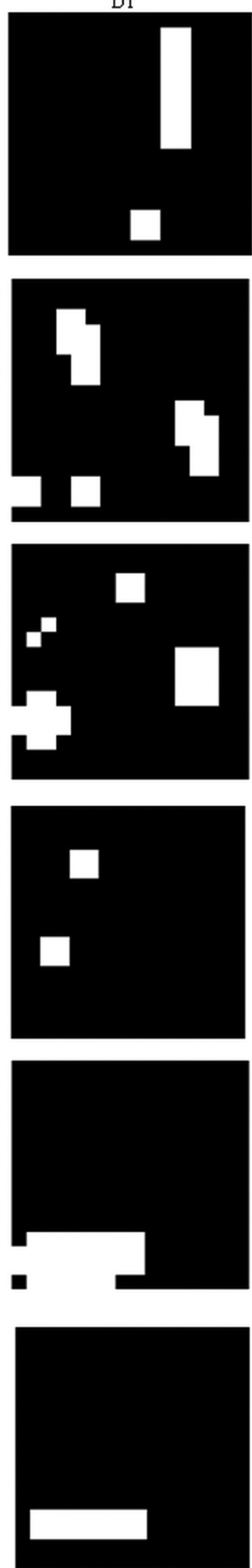

$R B$
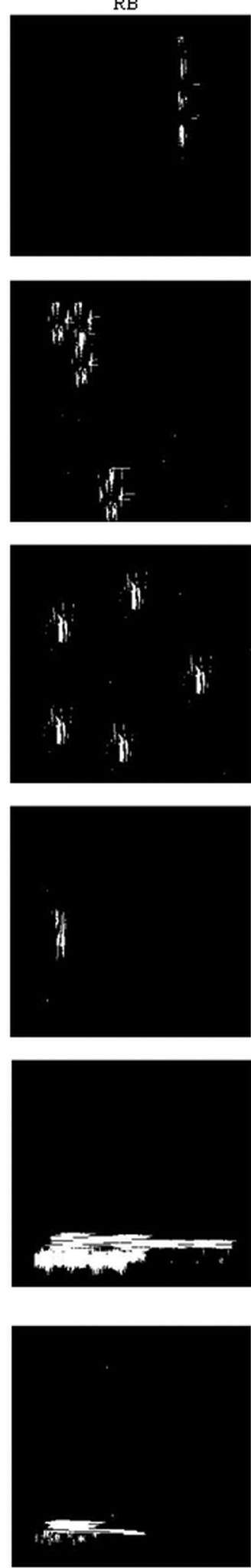

Fig. 11. Final results of methods of WGIS, DT and RB on different defective images: (a) broken end, (b) holes, (c) knots, (d) netting multiple, (e) thick bar, and (f) thin bar.

dard deviation [Fig. 4(1)]. If $u_{r_{n}}<\sigma_{r_{n}}, M=\sigma_{r_{n}}$, and this uses the value of one standard deviation as a measure for the severity of the defect.
3) Procedures of Regular Bands Method: The procedures of the RB method are as follows and the stages of the RB method is given in Fig. 5. 
Case (2)

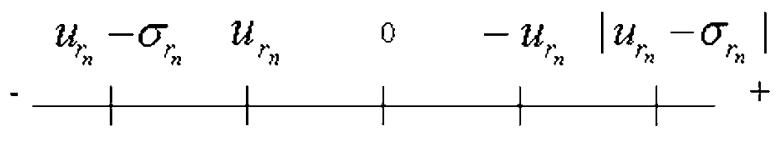

Fig. 12. Number line explains the proof of Lemma 1.

Training Stage:

Step 1. Histogram equalization of the input images.

Histogram equalization helps in reducing the noise on the images and makes the later threshold determination process more reliable.

Step 2. Down shifting of the images by the mean value of pixel intensities.

The process of downshifting on rows of both the LRB and DRB in the RB method are demonstrated in two defective samples in Fig. 4. Besides, how the LRB and DRB work on the defective rows of two defective images are shown in Fig. 4(k) and (1), respectively.

\section{Step 3. Calculation of the Regular bands.}

For every image, calculate the LRB and DRB on rows, then calculate both regular bands on columns. Fig. 6 graphically shows how the light regular band works on defect-free and defective samples of box-patterned fabric.

\section{Step 4. Obtain the threshold values.}

1. Calculate the light regular band and dark regular band on all rows and columns, respectively, for every $p \times q$ training defect-free image.

2. Obtain the upper bound and lower bound of the light regular band, $\left[l_{\min }^{i}, l_{\max }^{i}\right]$ and then obtain those of the dark regular band, $\left[d_{\min }^{i}, d_{\max }^{i}\right]$ after the calculations of the regular bands on row $i$. Average the upper bounds of the LRB on all rows $\overline{l^{i}}=\left[\sum_{i=1}^{p} l_{\mathrm{min}}^{k, i}\right] / p$, where $k$ is the $k$ th training sample. Then, for $f$ training samples, find the average of all upper bounds of the LRB, $l_{\min }=\left[\sum_{k=1}^{f} \overline{l^{k}}\right] / f$. Repeat the same process on the lower bounds of the light regular band and the lower and upper bounds of the dark regular band.

3. Four threshold values in two sets, $\left[l_{\min }, l_{\max }\right],\left[d_{\min }, d_{\max }\right]$ are obtained for the rows of the LRB and the DRB, respectively.

4. Repeat the same procedure described in 1-3 on columns and obtain another four threshold values.

Testing Stage:

Step 1. Histogram equalization of the input images.

Step 2. Shifting by the mean value of the defect-free images, $\bar{y}$.

Step 3. Calculate the Light and Dark Regular Bands on rows and columns of every image.
Step 4. Threshold the Regular Bands matrices with the corresponding threshold values determined during the training stage.

Step 5. Perform zero padding on the thresholded matrices.

Step 6. Combine the thresholded results of the LRB, $L^{\text {row }}$, and of the DRB, $D^{\text {row }}$, from the row of one image with an or-operation, $T^{\text {row }}=L^{\text {row }} \cup D^{\text {row }}$. Then, repeat the same step on the column side.

Step 7. Combine the results of rows and columns as a whole with an or-operation, $T=T^{\text {row }} \cup T^{\text {column }}$.

In the evaluation, every kind of fabrics used three defect-free image in the training stage and different numbers of images in the testing stage, 60, 50, and 56 images for the dot-patterned, star-patterned, and box-patterned fabrics, respectively.

4) Explanations of Steps in the RB Method:

Histogram Equalization: Defects on fabric are usually characterized by high frequency changes in pixel intensity within an image. Gaussian noise is usually found in the captured images and can cause a mix up with the real defects existing on the fabric. Also, the grey-scale range of pixel intensities is usually skewed to one side in the fabric image (Fig. 7). For this reason histogram equalization is used to obtain an even intensity distribution and a better contrast on the input images. It also helps to reduce the Gaussian noise from image acquisition, thus enabling the detection using regular bands to work more effectively. Fig. 8 shows a comparison of two defective samples with and without histogram equalization as a preprocessing step. The resultant images show better results with histogram equalized preprocessing than those without.

Two Lemmas of Regular Bands: With the step of down-shifting by the mean in one image, two lemmas apply.

Lemma 1: For the light regular band (LRB) on an image, the LRB is always greater than or equal to zero. That is $L_{r_{n}}=$ $\left|u_{r_{n}}-\sigma_{r_{n}}\right|+u_{r_{n}} \geq 0$ for $u_{r_{n}} \in R, \sigma_{r_{n}} \geq 0$ and $n \in Z^{+}$.

Proof: See the Appendix.

Lemma 2: For the dark regular band (DRB) on an image, the DRB is always greater than or equal to zero. That is $D_{r_{n}}=$ $\left|u_{r_{n}}+\sigma_{r_{n}}\right|-u_{r_{n}} \geq 0$ for $u_{r_{n}} \in R, \sigma_{r_{n}} \geq 0$ and $n \in Z^{+}$.

Proof: See the Appendix.

Effect Due to the Variation in the Period Length: There is a need to study the period length, $n$, which is the only variable parameter in the RB method. In the black star-patterned and blue box-patterned fabrics tested in our research, they are found to have repetitive units of size $25 \times 25$. Therefore, the period length of rows and columns are set to 25 as well. Table I shows the detailed detection results with period lengths of 24,25 , and 26 for regular bands on rows and columns, respectively, for the dot-patterned fabric database. Table II outlines the variations of $n$, with $n=10,15,22,23,24,25,26,27,28,30$, and 40 , for the detection results of the same database. Fig. 9 depicts the detection results of $n=10, n=15, n=25, n=30$, and $n=40$. In Table I, all the white-pixel numbers in the final thresholded images of defect-free samples cannot exceed 21 for $n=24, n=25$ and $n=26$. In Table II, the detection results 
TABLE I

Statistical Detection Results of 30 Defect-Free and 30 Defective Images of Dot-Patterned Fabric With $N=24,25$ and 26 , RESPECTIVELY, FOR REgUlAR BANDS ON ROWS AND COLUMNS

\begin{tabular}{|c|c|c|c|c|c|c|c|c|c|c|}
\hline \multirow{3}{*}{$\begin{array}{l}\text { Different } \\
\text { Images }\end{array}$} & \multicolumn{10}{|c|}{ Number of White Pixels in Final Threshold Images in the RB Method } \\
\hline & \multicolumn{4}{|c|}{$\mathbf{n}=\mathbf{2 4}$} & \multicolumn{3}{|c|}{$\mathbf{n}=\mathbf{2 5}$} & \multicolumn{3}{|c|}{$\mathrm{n}=\mathbf{2 6}$} \\
\hline & $\begin{array}{l}\text { Number of } \\
\text { Images }\end{array}$ & Mean & Max & Min & Mean & Max & Min & Mean & $\operatorname{Max}$ & Min \\
\hline Broken End & 5 & 1055.6 & 1196 & 259 & 970.60 & 2652 & 231 & 1194.4 & 3203 & 272 \\
\hline Hole & 5 & 914.80 & 1283 & 465 & 775 & 1052 & 403 & 1004 & 1443 & 497 \\
\hline Netting Multiple & 5 & 582.20 & 827 & 278 & 523.20 & 761 & 246 & 480 & 690 & 234 \\
\hline Knots & 5 & 845.60 & 1283 & 354 & 737.40 & 1061 & 305 & 643 & 915 & 275 \\
\hline Thick Bar & 5 & 6538.6 & 7630 & 4656 & 6449 & 7424 & 4635 & 6321.8 & 7248 & 4641 \\
\hline Thin Bar & 5 & 1563.2 & 2426 & 874 & 1485.6 & 2287 & 822 & 1400 & 2126 & 786 \\
\hline Defect-free & 30 & 5.23 & 16 & 0 & 2.67 & 11 & 0 & 4.83 & 21 & 0 \\
\hline
\end{tabular}

TABLE II

Mean Number of White Pixels in Final Threshold IMages (2 D.P.) OF 30 DefeCt-Free And 30 Defective IMAges of DOT-PATTERNED FABRIC IN THE RB METHOD FOR DifFERENT VALUES OF $N$

\begin{tabular}{|c|c|c|c|c|c|c|c|c|c|c|c|c|}
\hline $\begin{array}{l}\text { Different } \\
\text { Images }\end{array}$ & $\begin{array}{l}\text { No. of } \\
\text { Images }\end{array}$ & $\mathrm{n}=10$ & $\mathrm{n}=15$ & $\mathrm{n}=22$ & $\mathrm{n}=23$ & $n=24$ & $\mathrm{n}=25$ & $\mathrm{n}=26$ & $\mathrm{n}=27$ & $n=28$ & $\mathrm{n}=30$ & $\mathrm{n}=40$ \\
\hline Broken End & 5 & 54 & 185.6 & 665.6 & 935 & 1055.6 & 970.60 & 1194.4 & 1029.2 & 1013.6 & 984.8 & 1473 \\
\hline Hole & 5 & 186.4 & 381.6 & 581.2 & 802.6 & 914.80 & 775 & 1004 & 764.2 & 808.6 & 805.8 & 1419.2 \\
\hline Netting Multiple & 5 & 169 & 430.4 & 507.8 & 529.6 & 582.20 & 523.20 & 480 & 498.4 & 566.8 & 566.2 & 425 \\
\hline Knots & 5 & 147.4 & 288.2 & 596.4 & 693.8 & 845.60 & 737.40 & 643 & 713 & 884.2 & 912 & 868.4 \\
\hline Thick Bar & 5 & 4190.4 & 5027.6 & 5161.8 & 6101 & 6538.6 & 6449 & 6321.8 & 6095.2 & 6063.8 & 5904.2 & 5678.2 \\
\hline Thin Bar & 5 & 1242.6 & 1390.6 & 1294.2 & 1562 & 1563.2 & 1485.6 & 1400 & 1298 & 1260.4 & 1146.4 & 944.4 \\
\hline Defect-free & 30 & 2.47 & 2.63 & 2.3 & 3.87 & 5.23 & 2.67 & 4.83 & 4.67 & 5.37 & 4.67 & 6.37 \\
\hline
\end{tabular}

perform similarly from $n=22$ to $n=30$. Also, from Tables I and II, the detection boundaries of the amount of white pixels in the final thresholded results between defect-free and defective images are still very clear. In Fig. 9, the detection result of $n=$ 25 is subjectively outstanding while the results of $n=15$ and $n=30$ are still acceptable. Therefore, it can be concluded that the period length, $n$, of the regular bands is not sensitive to a slight variation.

\section{Evaluation Using the Regular Bands Method on PATTERNED FABRICS}

\section{A. Detection Results of the RB Method}

In this section, two sets of patterned fabrics (star-patterned and box-patterned) are inspected using the method of regular bands. There are totally 106 including 50 defect-free and 56 defective samples with five types of defects. Every image is of size $256 \times 256$ in grey-scale. The computer used for the inspection is a Pentium $43.2 \mathrm{GHz}, 1 \mathrm{~GB}$ DDR RAM. The algorithm is implemented in MATLab (version 7).

The regular bands method is novel since it only requires the determination of one parameter, the length of period of the repetitive unit in the patterned texture. The star-patterned and box-patterned fabric evaluated in this paper has the same period length $(n)$ of 25 for both rows and columns. A resultant image from the RB process will be slightly smaller than the original size due to the row and column dimensions of the repetitive unit that is used in the calculation of the RB. Zero padding has modified the detection result so that a visual comparison of final results can be more easily judged.
TABLE III

Statistical DeteCtion Results of Defect-Free IMAges IN Two DIFFERENT PATTERNED FABRICS

\begin{tabular}{cccccc}
\hline \hline $\begin{array}{c}\text { Number of White Pixels } \\
\text { in Final Threshold } \\
\text { Images }(\mathbf{n}=25)\end{array}$ & $\begin{array}{c}\text { Number of } \\
\text { Images }\end{array}$ & Mean & Max & Min & $\begin{array}{c}\text { Standard } \\
\text { Deviation }\end{array}$ \\
\hline Black Star-patterned & 25 & 3.32 & 14 & 6 & 4.18 \\
\hline Blue Box-patterned & 30 & 43.43 & 111 & 0 & 36.36 \\
\hline \hline
\end{tabular}

The thresholded image is of size $232 \times 232$ (53 824 pixels). A defective image is defined as an image with a considerable number of pixels exceeding the normal range defined by the regular bands. They appear as white pixels after thresholding in the RB method. So, a final thresholded image is determined to be defective if it exceeds a certain amount of white pixels out of 53824 pixels. Three statistical tables of detection results are given in Tables III-V. In the testing stage, the maximum number of white pixels in the final threshold images for the defect-free star-patterned and box-patterned samples are 14 and 111, respectively. The average number of white pixels among the training samples is 3.3 and 43.3 for star-patterned fabric and box-patterned fabrics, respectively. From a set of defect-free samples used in the training stage, the threshold values are then set as $30(0.056 \%)$ and $120(0.23 \%)$ white pixels out of 53834 for star-patterned fabric and box-patterned fabric, respectively. For star-patterned fabric, the detection success rate is $100 \%$ for 25 defect-free images and 25 defective images. For box-patterned fabric, the detection success rate is $100 \%$ for 30 defectfree images and $96.15 \%$ for 26 defective images ( 1 defective 
TABLE IV

STATISTICAL DETECTION RESUltS OF 25 IMAGES OF BLACK STAR-PATTERNED FABRIC

\begin{tabular}{cccccc}
\hline \hline & \multicolumn{4}{c}{$\begin{array}{c}\text { Number of White Pixels in Final Threshold Images } \\
\mathrm{n}=25 \text { in RB on Row and Column }\end{array}$} \\
\cline { 2 - 6 } $\begin{array}{c}\text { Different Kinds } \\
\text { of Defective } \\
\text { Images }\end{array}$ & $\begin{array}{c}\text { Number of } \\
\text { Images }\end{array}$ & Mean & Max & Min & $\begin{array}{c}\text { Standard } \\
\text { Deviation }\end{array}$ \\
\hline Broken End & 5 & 282 & 800 & 70 & 265.35 \\
\hline Hole & 5 & 103.6 & 162 & 39 & 41.73 \\
\hline Netting Multiple & 5 & 372.6 & 933 & 98 & 297.69 \\
\hline Thick Bar & 5 & 687.2 & 2127 & 69 & 238.91 \\
\hline Thin Bar & 5 & 74.2 & 101 & 44 & 18.48 \\
\hline \hline
\end{tabular}

TABLE V

StATisticAl Detection Results of 26 IMAGES OF BLUE BOX-PATTERNED FABRIC

\begin{tabular}{lccccc}
\hline \hline & \multicolumn{4}{c}{$\begin{array}{c}\text { Number of White Pixels in Final Threshold Images } \\
\mathrm{n}=25 \text { in RB on Row and Column }\end{array}$} \\
\cline { 2 - 6 } $\begin{array}{c}\text { Different Kinds } \\
\text { of Defective } \\
\text { Images }\end{array}$ & $\begin{array}{c}\text { Number of } \\
\text { Images }\end{array}$ & Mean & Max & Min & $\begin{array}{c}\text { Standard } \\
\text { Deviation }\end{array}$ \\
\hline Broken End & 5 & 1064.2 & 1309 & 906 & 143.78 \\
\hline Hole & 5 & 132 & 179 & 77 & 34.13 \\
\hline Netting Multiple & 5 & 278.8 & 640 & 148 & 182.62 \\
\hline Thick Bar & 6 & 1994 & 3747 & 436 & 1345.80 \\
\hline Thin Bar & 5 & 488.2 & 700 & 145 & 204.86 \\
\hline \hline
\end{tabular}

sample failed to be detected). Thus, the overall detection success rate of this fabric is $98.21 \%$ (55/56). Finally, the overall detection success rate of these two fabrics is $99.1 \%$ (110/111). The detection results of each class of defective samples are shown in Fig. 10.

\section{B. A Comparison of the Inspection Results for the WGIS, DT and RB Methods}

In [11], there is a 60-image database of a dot-patterned Jacquard fabric with defective and defect-free samples. For the 60-image database, the WGIS method showed a high overall detection success rate, i.e., 96.7\% [11]. All defective images were correctly detected and only two defect-free images were misclassified. Each final image (the left-most column of Fig. 11) including the six types of defects could be outlined by the WGIS method. However, the general perception for each final image in the WGIS method is that the white areas (the defective regions) would show an enlarged version of the actual defective parts in the original defective images. For example, for thick bar (Tk1) and thin bar (Tn1), the final images of the WGIS method are rounded in shape, magnified in size and shifted a little bit in position compared with that of the RB method. This is mainly due to the effect of golden image subtraction in the WGIS method since the golden image is larger than one repetitive-unit size, thus acting like a convolution filter on the input image. It results in the borders of the subtracted image having a padding effect (similar to effect of an actual convolution on an image) with extended defective areas on the final image.

Compared with the WGIS and RB methods, the method of DT depicted coarser results. The final images of six types of defects (third column in Fig. 11) delivered rectangular shape results due to low resolution of detailed subimages $(16 \times 16$ in fourth-level Haar wavelet decomposition) through the multiresolution property of a wavelet transform. Excessive noise in final images was commonly found, such as the final images of broken end (B1) and holes (H1). The coarser results and excessive noise would actually be quite confusing to some extent if the classification of defects had been implemented as a whole. The overall detection success rate was $88.3 \%$ [11] for a 60 -image set.

Using the statistical techniques of moving average and standard deviation, the method of RB has generated much better results than the two previous methods. Not only are the final images of the six typical types of defects clean and crystal clear, especially for thick bar (Tk1) and thin bar (Tn1), but the overall detection success rate of $100 \%$ was the highest among the three methods, achieved on the same image database above. The detection results are shown in the fourth column of Fig. 11. This result was robust and satisfactory for dot-patterned Jacquard fabric defect detection. A general comparison of the three methods is given in Table VI.

\section{DISCUSSIONS}

The overall defect detection success rate of the RB method is $99.4 \%$ for three patterned fabrics with 166 images. A new principle for the measurement of regularity is evaluated in this paper. The intensities of any one row or column, which can be called a row signal (or a column signal), would in general be characterized by a periodic waveform regardless of its pattern texture. Through particular transforms (GIS, DT, or RB) on these periodic intensities in rows or columns, the resultant signal will also have a periodic waveform. The aim of the transformation from one periodic signal to a new one is to enhance the irregularity, or defective information, on the new signal. For examples, the WGIS method uses subtractions to enhance the defective regions. The DT method applies wavelet transforms to generate horizontal and vertical details for localizing the defective information. The RB method enhances the defective regions through the calculations of moving averages and standard deviations. The enhancement of defective regions would appear to be subtle changes when compared with the normal transformed signal, but after a transformation of signals thresholding on the new signal can segment out the defects. The extension from a one-dimensional signal into two-dimensional signals (as an image) enables the consistent detection of defects.

\section{CONCLUSION}

After a review of the various approaches to regularity analysis for patterned textures, it becomes apparent that the RB method is a new and effective approach for patterned texture inspection. The RB method can detect various kinds of defects and outline their shapes. This information will be useful for defect recognition for pattern classification in further research. In comparison, not all of the traditional approaches to periodicity can be used to segment the defects and outline their shapes. A minor problem of the RB method is its inability to detect the defects on the borders of image due to the definition of RB, with a period length of data as reference. The strengths of RB are as follows.

The Regular Bands are shift-invariant and statistically sensitive to abnormal parts. The method is effective for segmenting 
TABLE VI

COMPARISON OF METHODS OF WGIS, DT AND RB

\begin{tabular}{|c|c|c|c|c|c|}
\hline $\begin{array}{l}\text { Method } \\
\text { (Main Idea) }\end{array}$ & Dimensionality & $\begin{array}{c}\text { Ability to } \\
\text { Outline the } \\
\text { Defective Shape }\end{array}$ & $\begin{array}{l}\text { Detection Success } \\
\text { Rate on } 60 \text { Dot- } \\
\text { Patterned Images }\end{array}$ & Strengths & Weaknesses \\
\hline $\begin{array}{l}\text { The WGIS } \\
\text { Method }\end{array}$ & 2 & Good & $96.7 \%$ & $\begin{array}{l}\text { 1. Easy to choose the size of Golden } \\
\text { Image } \\
\text { 2. Shortest time complexity among all } \\
\text { methods in implementation }\end{array}$ & $\begin{array}{c}\text { Cannot detect defect near } \\
\text { the borders }\end{array}$ \\
\hline The DT Method & 2 & Poor & $88.3 \%$ & $\begin{array}{l}\text { Haar wavelet transformed subimages } \\
\text { are lower resolution so that lower } \\
\text { computational power in detection part. }\end{array}$ & $\begin{array}{l}\text { Coarse in detection } \\
\text { results }\end{array}$ \\
\hline The RB Method & 1 & Excellent & $100 \%$ & $\begin{array}{l}\text { 1. Simplicity : RB is built on the concept } \\
\text { of moving average and standard } \\
\text { deviation }\end{array}$ & $\begin{array}{c}\text { Cannot detect defect near } \\
\text { the borders }\end{array}$ \\
\hline
\end{tabular}

Case $\{2)$

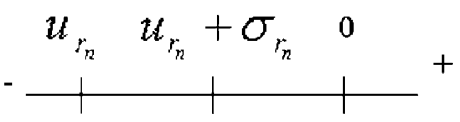

Fig. 13. Number line explains the proof of Lemma 2

out and outlining the shape of the defective regions. It is sensitive to small defects provided there is sufficient change in intensity, and the final thresholded image is clean. The method is easy to use and implement. It only requires knowledge of the period length of a repetitive pattern, and the method is also not overly sensitive to this parameter. Image alignment or distortion problems due to the camera not being perfectly perpendicular or parallel to the fabric do not seem to weaken the method. The time needed for detection of an image of size $256 \times 256$ is $15.663 \mathrm{~s}$ when implemented using MATLAB. However, when implemented using the $\mathrm{C}$ programming language, the computational time is only $140 \mathrm{~ms}$. Hence, the RB method is fast enough for real-time on-loom defect detection.

\section{APPENDIX}

\section{PROOFS OF LEMMA 1 AND LEMMA 2}

Proof of Lemma 1: Case (1). For $u_{r_{n}} \geq 0$ and $\sigma_{r_{n}} \geq 0$, in $L_{r_{n}},\left|u_{r_{n}}-\sigma_{r_{n}}\right| \geq 0$ and $u_{r_{n}} \geq 0$, so that $\left|u_{r_{n}}-\sigma_{r_{n}}\right|+u_{r_{n}} \geq 0$. Case (2). For $u_{r_{n}}<0$ and $\sigma_{r_{n}} \geq 0,\left|u_{r_{n}}-\sigma_{r_{n}}\right| \geq-u_{r_{n}} \Leftrightarrow$ $\left|u_{r_{n}}-\sigma_{r_{n}}\right|+u_{r_{n}} \geq 0$. Therefore, $L_{r_{n}}=\left|u_{r_{n}}-\sigma_{r_{n}}\right|+u_{r_{n}} \geq 0$ for $u_{r_{n}} \in R, \sigma_{r_{n}} \geq 0$ and $n \in Z^{+}$.

Fig. 12 illustrates the concept of the Case (2) of proof of Lemma 1.

Proof of Lemma 2: Case (1). For $u_{r_{n}} \geq 0$ and $\sigma_{r_{n}} \geq 0$ in $D_{r_{n}}, u_{r_{n}}+\sigma_{r_{n}} \geq u_{r_{n}}$, so that $\left|u_{r_{n}}+\sigma_{r_{n}}\right| \geq u_{r_{n}} \Leftrightarrow$ $\left|u_{r_{n}}+\sigma_{r_{n}}\right|-u_{r_{n}} \geq 0$. Case (2). For $u_{r_{n}}<0$ and $\left|u_{r_{n}}\right| \geq u_{r_{n}}$, $\left|u_{r_{n}}+\sigma_{r_{n}}\right| \geq\left|u_{r_{n}}\right| \geq u_{r_{n}}$, which is equivalent to $\left|u_{r_{n}}+\sigma_{r_{n}}\right|-$ $u_{r_{n}} \geq 0$. Therefore, $D_{r_{n}}=\left|u_{r_{n}}+\sigma_{r_{n}}\right|-u_{r_{n}} \geq 0$ for $u_{r_{n}} \in R$, $\sigma_{r_{n}} \geq 0$ and $n \in Z^{+}$.

Fig. 13 illustrates the concept of the Case (2) of proof of Lemma 2.

\section{ACKNOWLEDGMENT}

The authors would like to thank K. Senechal for proofreading the paper.

\section{REFERENCES}

[1] M. Tuceryan and A. K. Jain, "Texture analysis," in Handbook of Pattern Recognition \& Computer Vision, 2nd ed. Hackensack, NJ: World Scientific, 1999, pp. 207-248.

[2] L. G. Shapiro and G. C. Stockman, Computer Vision. Englewood Cliffs, NJ: Prentice-Hall, 2001.

[3] R. Jain, R. Kasturi, and B. G. Schunck, Machine Vision. New York: McGraw-Hill, 1995.

[4] K. I. Laws, "Texture Image Segmentation," Ph.D. dissertation, Univ. Southern California, Los Angeles, 1980.

[5] D. Chetverikov, "Pattern regularity as a visual key," Image Vis. Comput., vol. 18, pp. 975-985, 2000.

[6] D. Chetverikov and A. Hanbury, "Finding defects in texture using regularity and local orientation," Pattern Recognit., vol. 35, pp. 2165-2180, 2002.

[7] H. C. Lin, L. L. Wang, and S. N. Yang, "Regular-texture image retrieval based on texture-primitive extraction," Image Vis. Comput., vol. 17, pp. 51-63, 1999.

[8] C. F. J. Kuo and T. L. Su, "Gray relational analysis for recognizing fabric defects," Textile Res. J., vol. 73, no. 5, pp. 461-465.

[9] L. G. C. Hamey and T. Kanade, "Computer analysis of regular repetitive textures," in Proc. DARPA Image Understanding Workshop, 1989 , pp. 1076-1088.

[10] Y. Liu, W. C. Lin, and J. Hayes, "Near-regular texture analysis and manipulation," ACM Trans. Graphics (SIGGRAPH), vol. 23, no. 3, pp. 368-376, 2004.

[11] H. Y. T. Ngan, G. K. H. Pang, S. P. Yung, and M. K. Ng, "Wavelet based methods on patterned fabric defect detection," Pattern Recognit., vol. 38, no. 4, pp. 559-576, 2005.

[12] C. Sandy, L. Norton-Wayne, and R. Harwood, "The automated inspection of lace using machine vision," Mech. J., vol. 5, no. 2/3, pp. 215-231, 1995

[13] I. C. Baykal, R. Muscedere, and G. A. Jullien, "On the use of hash functions for defect detection in textures for in-camera web inspection systems," in Proc. IEEE Int. Symp. Circuits Systems, ISCAS, 2002, vol. 5, pp. 665-668.

[14] I. C. Baykal and G. A. Jullien, "Detection of defects in textures with alignment error for real-time line-scan web inspection systems," in Proc. IEEE MWSCAS The 2002 45th Midwest Symp. Circuits Systems, Aug. 4-7, 2002, vol. 3, pp. 292-295.

[15] L. M. Hoffer, F. Francini, B. Tiribilli, and G. Longobardi, "Neural network for the optical recognition of defects in cloth," Opt. Eng., vol. 35, no. 11, pp. 3183-3190, Nov. 1996

[16] I. S. Tsai, C. H. Lin, and J. J. Lin, "Applying an artificial neural network to pattern recognition in fabric defects," Textile Res. J., vol. 65, no. 3, pp. 123-130, 1995.

[17] C. H. Chan and G. K. H. Pang, "Fabric defect detection by Fourier analysis," IEEE Trans. Ind. Applicat., vol. 36, no. 5, pp. 1267-1276, Sep./Oct. 2000

[18] E. J. Wood, "Applying Fourier and associated transforms to pattern characterization in textiles," Textile Res. J., vol. 60, pp. 212-220, 1990. 
[19] A. Kumar and G. Pang, "Defect detection in textured materials using Gabor filters," IEEE Trans. Ind. Applicat., vol. 38, no. 2, pp. 425-440, Mar./Apr. 2002.

[20] A. Bodnarova, M. Bennamoun, and S. Latham, "Optimal Gabor filters for textile flaw detection,” Pattern Recognit., vol. 35, pp. 2973-2991, 2002.

[21] D. M. Tsai and S. K. Wu, "Automated surface inspection using Gabor filters," Int. J. Adv. Manufact. Technol., vol. 16, pp. 474-482, 2000.

[22] G. Lambert and F. Bock, "Wavelet methods for texture defect detection," in Proc. IEEE Int. Conf. Image Processing, 1997, vol. 3, pp. $26-29$.

[23] A. L. Amet, A. Ertuzun, and A. Ercil, "An efficient method for texture defect detection: Sub-band domain co-occurrence matrices," Image Vis. Comput., vol. 18, pp. 543-553, 2000.

[24] X. Z. Yang, G. K. H. Pang, and N. Yung, "Robust fabric defect detection and classification using multiple adaptive wavelets," Proc. Inst. Elect. Eng., vol. 152, no. 6, Dec. 2005.

[25] R. T. Chin and C. A. Harlow, "Automated visual inspection: A survey," IEEE Trans. Pattern Anal. Machine Intell., vol. PAMI-4, no. 6, pp. 557-573, Nov. 1982.

[26] M. L. Smith and R. J. Stamp, "Automated inspection of textured ceramic tiles," Comput. Ind., vol. 43, pp. 73-82, 2000.

[27] R. M. Haralick and L. G. Shapiro, Computer and Robot Vision. Reading, MA: Addison-Wesley, 1991, vol. 1.

[28] L. Tao, P. Witty, and T. King, "Machine vision in the inspection on patterned textile webs," IEE Colloq., pp. 9/1-9/5, Ind. Inspect. (Digest No: 1997/041).

[29] M. S. Millan and J. Escofet, "NIR imaging of non-uniform colored webs: Application to fabric inspection," Proc. SPIE, vol. 5622, pp. 188-193, 2004.

[30] H. Y. T. Ngan, G. K. H. Pang, S. P. Yung, and M. K. Ng, "Defect detection on patterned Jacquard fabric," in Proc. IEEE Int. Workshop on 32nd Applied Imagery Pattern Recognition, Oct. 2003, pp. 163-168.

[31] H. Y. T. Ngan and G. K. H. Pang, "Novel method for patterned fabric inspection using Bollinger bands," Opt. Eng., vol. 45, no. 8, Aug. 2006.

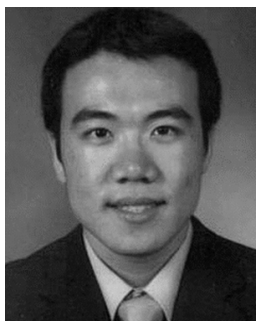

Henry Y. T. Ngan (S'02) received the B.Sc. degree in mathematics in 2001, and the M.Phil. degree in electrical and electronic engineering in 2004 from the University of Hong Kong, China. He is currently pursuing the Ph.D. degree in the Industrial Automated Research Laboratory, Department of Electrical and Electronic Engineering, University of Hong Kong.

He was a Visiting Scholar with the Industrial Automated Laboratory, University of British Columbia, B.C., Canada, from March to June of 2006. His current research topic is the Inspection of patterned textures and materials and his research interests include surface defect detection, image processing, machine intelligence, pattern recognition, and computer vision.

Mr. Ngan is a student member of the IET.

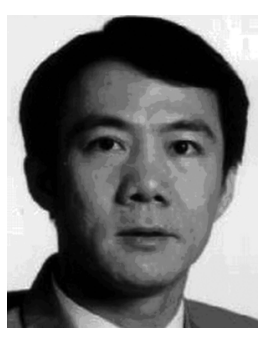

Grantham K. H. Pang (S'84-M'86-SM'01) received the Ph.D. degree from the University of Cambridge, Cambridge, U.K., in 1986.

He was with the Department of Electrical and Computer Engineering, University of Waterloo, Waterloo, ON, Canada, from 1986 to 1996. Currently, he is an Associate Professor with the Department of Electrical and Electronic Engineering, University of Hong Kong, in 1996. Since 1988, he published more than 150 technical papers and has authored or co-authored six books. He has also obtained five U.S. patents. His research interests include machine vision for surface defect detection, optical communications, expert systems for control system design, intelligent control, and intelligent transportation systems.

Dr. Pang is a Chartered Electrical Engineer, and a member of the IET and HKIE. In 1989, he was awarded the ICI Prize for authorship of the best paper on the application of the theory of control published in the Transactions of Institute of Measurement and Control. 